\title{
Betweenness centrality correlation in social networks
}

\author{
K.-I. Goh, E. Oh, B. Kahng, and D. Kim \\ School of Physics and Center for Theoretical Physics, Seoul National University, Seoul 151-747, Korea
}

(Received 11 October 2002; published 13 January 2003)

\begin{abstract}
Scale-free (SF) networks exhibiting a power-law degree distribution can be grouped into the assortative, dissortative, and neutral networks according to the behavior of the degree-degree correlation coefficient. Here we investigate the betweenness centrality (BC) correlation for each type of SF networks. While the BC-BC correlation coefficients behave similarly to the degree-degree correlation coefficients for the dissortative and neutral networks, the $\mathrm{BC}$ correlation is nontrivial for the assortative ones found mainly in social networks. The mean $\mathrm{BC}$ of neighbors of a vertex with $\mathrm{BC} g_{i}$ is almost independent of $g_{i}$, implying that each person is surrounded by almost the same influential environments of people no matter how influential the person may be.
\end{abstract}

DOI: 10.1103/PhysRevE.67.017101

PACS number(s): 89.75. $-\mathrm{k}, 89.20 .-\mathrm{a}, 89.65 .-\mathrm{s}$

Recently there have been considerable efforts to understand complex systems in terms of random graph, consisting of vertices and edges, where vertices (edges) represent constituents (their interactions) [1,2]. An interesting feature emerging in such complex networks is a power-law degree distribution, $p_{d}(k) \sim k^{-\gamma}$, where the degree $k$ is the number of edges incident upon a given vertex $[3,4]$. Networks displaying the power-law degree distribution are called the scale-free (SF) networks. Barabási and Albert (BA) introduced an evolving model illustrating such a SF network [5]. The degree distribution of the BA model follows a powerlaw $p_{d}(k) \sim k^{-3}$. While the BA-type models are meaningful as the first step to generate SF network, they are too simple to accord with real-world networks, exhibiting nontrivial degree-degree and other correlations.

The SF networks can be grouped into three types according to the behavior of the degree-degree correlation coefficient $[6,7]$. They are those exhibiting the assortative, dissortative, and neutral mixing on their degree. For the network of the assortative (dissortative) mixing, called the assortative (dissortative) network, a vertex with large degree tends to connect to vertices with large (small) degree, while for the network of the neutral mixing, there is no such tendency. The assortative network can be found in social networks such as the coauthorship network, the actor network and so on, and the dissortative network in information networks such as the internet and the World Wide Web, and in biological networks such as protein interaction networks and neural networks. While such assortative and dissortative networks appear in real world, the neutral network, i.e., the network of the neutral mixing on their degree, appears in in silico networks such as the BA model and the copying model [8] with $\gamma$ $=3$.

While degree is a fundamental quantity describing the topology of the SF network, it was shown that the BC is another important quantity to characterize how influential a vertex is in communications between each pair of vertices $[9,10]$. To be specific, let us suppose communication paths between a pair of vertices $(i, j)$ are the shortest pathways and let the number of such pathways denoted by $c(i, j)$. Among them, the number of the shortest pathways running through a vertex $k$ is denoted by $c_{k}(i, j)$ and the fraction by $g_{k}(i, j)$ $=c_{k}(i, j) / c(i, j)$. Then the BC of the vertex $k$ is defined as the accumulated amount of $g_{k}(i, j)$ over all pairs, i.e., $g_{k}$ $=\sum_{\{(i . j)\}} c_{k}(i, j) / c(i, j)=\sum_{\{(i, j)\}} g_{k}(i, j)$. The BC distribution follows a power law for SF networks, $p_{b}(g) \sim g^{-\eta}$, where $g$ means BC and the exponent $\eta$ turns out to be robust as either $\eta \approx 2.2$ or $\eta=2.0$, independent of the degree exponent as long as $2<\gamma \leqslant 3[11,12]$.

For the BA-type model, it was shown that the $\mathrm{BC}$ is related to the degree via the relation [11]

$$
g \sim k^{(\gamma-1) /(\eta-1)}
$$

Thus the vertices with larger degree are much more influential to others in communications. Due to this relation, one may think that the BC-BC correlation would behave similarly to the degree-degree correlation. In this paper, we report that while for the dissortative and neutral network, the BC-BC correlation coefficients behave similarly to the degree-degree correlation coefficients, for the assortative network, the degree-BC relation Eq. (1) is nontrivial, leading to that the $\mathrm{BC}-\mathrm{BC}$ correlation is very weakly assortative, i.e., the mean BC of neighbors of a certain vertex with BC $g_{i}$ is almost independent of $g_{i}$.

The degree-degree correlation $[13,14]$ was investigated in terms of the correlation function between the remaining degrees of the two vertices on each side of an edge, where the remaining degree means the degree of that vertex minus one [6]. First one defines the joint probability $e_{d}(j, k)$ that the two vertices on each side of a randomly chosen link have $j$ and $k$ remaining degrees, respectively. Then the normalized correlation coefficient is defined as

$$
r_{d}=\frac{1}{\sigma_{d}(q)^{2}} \sum_{j, k} j k\left\{e_{d}(j, k)-q_{d}(j) q_{d}(k)\right\},
$$

where $q_{d}(k)$ is the normalized distribution of the remaining degree $q_{d}(k)=(k+1) p_{d}(k+1) / \Sigma_{j} j p_{d}(j), \quad$ and $\quad \sigma_{d}(q)^{2}$ $=\Sigma_{k} k^{2} q_{d}(k)-\left[\Sigma_{k} k q_{d}(k)\right]^{2}$. Recently Newman called this quantity the degree assortativity coefficient [7]. For the assortative (dissortative) networks, $r_{d}$ is positive (negative), and for the neutral networks, $r_{d}=0$. On the other hand, the degree-degree correlation was also investigated in terms of the mean degree of neighbors of a vertex with degree $k$, denoted by $\left\langle k_{\mathrm{nn}}\right\rangle(k)$ [15]. For the assortative (dissortative) 

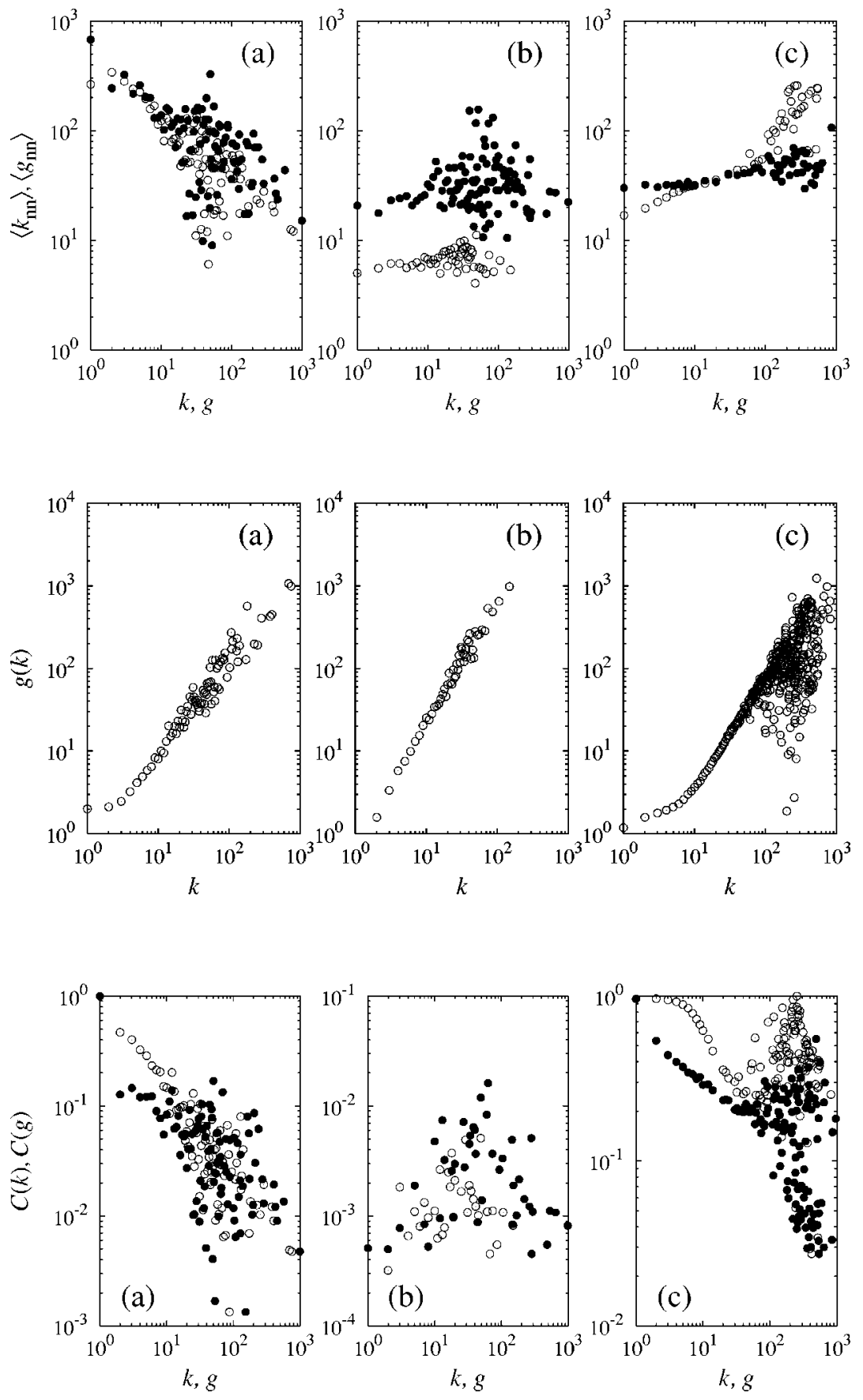

FIG. 1. Plot of $\left\langle k_{\mathrm{nn}}\right\rangle(k)(\bigcirc)$ and $\left\langle g_{\mathrm{nn}}\right\rangle(g)$ (O) for (a) the Internet on the level of autonomous systems (dissortative), (b) the nondegenerate configuration model with $\gamma=3$ (neutral), and (c) the coauthorship network in the field of neuroscience (assortative). All data are obtained from a single configuration.

FIG. 2. Plot of the degree-BC relation for (a) the Internet on the level of autonomous systems (dissortative), (b) the nondegenerate configuration model with $\gamma=3$ (neutral), and (c) the coauthorship network in the field of neuroscience (assortative).

TABLE I. Size $N$, mean degree $\langle k\rangle$, degree assortativity coefficient $r_{d}$, BC assortativity coefficient $r_{b}$ for a number of social networks.

\begin{tabular}{lcccccc}
\hline \hline Type & Name & $N$ & $\langle k\rangle$ & $r_{d}$ & $r_{b}$ & Reference \\
\hline \multirow{4}{*}{ Actor } & Videomovie & 29824 & 33.7 & 0.22 & 0.024 & {$[19]$} \\
& TVminiseries & 33980 & 73.0 & 0.38 & 0.033 & {$[19]$} \\
& TVcablemovies & 117655 & 55.5 & 0.14 & 0.035 & {$[19]$} \\
& TVseries & 79663 & 118.4 & 0.53 & 0.013 & {$[19]$} \\
Coauthor & Neuroscience & 205202 & 11.8 & 0.60 & 0.057 & {$[20]$} \\
& Mathematics & 78835 & 5.50 & 0.59 & 0.091 & {$[20]$} \\
& cond-mat & 16264 & 5.85 & 0.18 & 0.086 & {$[21]$} \\
& arXiv.org & 52909 & 9.27 & 0.36 & 0.057 & {$[21]$} \\
\hline \hline
\end{tabular}

FIG. 3. Plot of the clustering coefficient as a function of degree $(\mathrm{O})$ and $\mathrm{BC}(\boldsymbol{O})$ for $(\mathrm{a})$ the Internet on the level of autonomous systems (dissortative), (b) the nondegenerate configuration model with $\gamma=3$ (neutral), and (c) the coauthorship network in the field of neuroscience (assortative). 


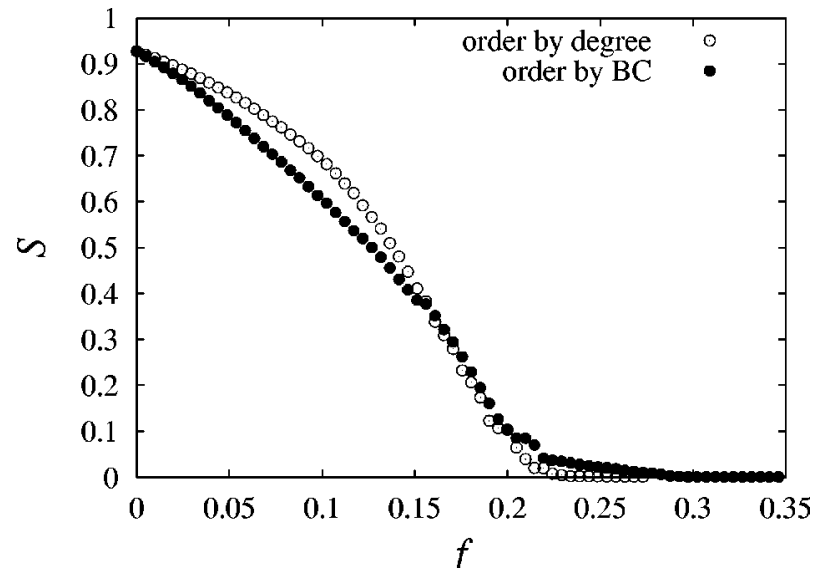

FIG. 4. Plot of the relative size $S$ of the giant cluster as a function of the fraction $f$ of removed vertices following the order of degree $(\bigcirc)$ and $\mathrm{BC}(\bigcirc)$ for the coauthorship network in the field of neuroscience (assortative).

networks, $\left\langle k_{\mathrm{nn}}\right\rangle(k)$ increases (decreases) with increasing $k$, while the neutral networks, $\left\langle k_{\mathrm{nn}}\right\rangle(k)$ is independent of $k$.

To study the BC-BC correlation, we introduce the $\mathrm{BC}-\mathrm{BC}$ correlation coefficient, called the $\mathrm{BC}$ assortativity coefficient, in analogy with Eq. (2) as

$$
r_{b}=\frac{1}{\sigma_{b}(q)^{2}} \sum_{\ell, m} \ell m\left\{e_{b}(\ell, m)-p_{b}(\ell) p_{b}(m)\right\},
$$

where $e_{b}(\ell, m)$ is the joint probability that the BCs of the two vertices of a link are $\ell$ and $m$ and $\sigma_{b}(q)^{2}=\Sigma_{\ell} \ell^{2} p_{b}(\ell)$ $-\left[\Sigma_{\ell} \ell p_{b}(\ell)\right]^{2}$. Moreover, similarly to $\left\langle k_{\mathrm{nn}}\right\rangle$, we define the mean $\mathrm{BC}$ of neighbors of a vertex with $\mathrm{BC} g$, denoted by $\left\langle g_{\mathrm{nn}}\right\rangle(g)$, through which we can check if the BC-BC correlation is assortative or dissortative.

We first check the $\mathrm{BC}-\mathrm{BC}$ correlation for the network of the Internet on the level of autonomous systems as of January 2000 [16] and the so-called nondegenerate configuration model with $\gamma=3[14,17,18]$, which belong to the dissortative and the neutral network, respectively. For these networks, $r_{b}$ is $-0.16(<0)$ and 0.02 , respectively, which is close to their $r_{d}$ values of -0.18 and 0.01 , respectively. Moreover, $\left\langle g_{\mathrm{nn}}\right\rangle$ $\times(g)$ behaves similarly to $\left\langle k_{\mathrm{nn}}\right\rangle(k)$ as shown in Fig. 1(a) and 1(b). However for the assortative networks, the coauthorship network for example, $r_{b}$ is considerably smaller than $r_{d}$ often by one order of magnitude and is close to zero. The comparison of $r_{b}$ and $r_{d}$ for various social networks are tabulated in Table I. The mean BC $\left\langle g_{\mathrm{nn}}\right\rangle(g)$ of neighbors of a vertex with BC $g$ increases with increasing $g$, however, the increasing rate is very low compared with that of $\left\langle k_{\mathrm{nn}}\right\rangle(k)$, i.e., it depends on $g$ very weakly [Fig. 1(c)]. Such a behavior appears in other social networks too. Since BC is regarded as a good measure of centrality, it implies that the mean influence of neighbors of a person is almost the same regardless of the influence of the centered person. So a person is surrounded by almost the same influential people on average no matter how influential the centered person is, although a person who acquaints many people is likely to connect to people who also acquaint many others.

To understand the abnormal behavior of the $\mathrm{BC}-\mathrm{BC}$ correlation in detail, we examine the degree-BC relation. In Fig. 2 , we compare the degree- $\mathrm{BC}$ relation $g(k)$ for the three types. While the relation of Eq. (1) holds for the dissortative and the neutral networks, it breaks down for large $k$ for the assortative networks. Rather the BCs of large $k$ vertices cover wide range of values. Since the vertices with large degree are located next to each other in the assortative network, the shortest pathways between a certain pair of vertices do not necessarily pass through such nearby hubs at the same time. Thus the BCs of the vertices with large $k$ fluctuate and the degree-BC correlation is nontrivial. Next, we compare the clustering coefficient $C(k)$ as a function of degree $k$ with that $C(g)$ as a function of $\mathrm{BC} g$ in Fig. 3. Again for the assortative and the neutral network, the two functions almost overlap, however, for the assortative network, the two functions are distinct.

To compare the contributions of degree and $\mathrm{BC}$ to the robustness of networks, we also study the relative size of the giant cluster $S$ as a function of the fraction of removed vertices $f$ [22]. We measure $S$ in the two ways of vertex removal following the order of (i) degree and (ii) $\mathrm{BC}$ for an assortative network. Figure 4 shows the data for the coauthorship network in the neuroscience field. The relative size $S$ by the vertex removal in $\mathrm{BC}$ order decreases faster than in degree order up to $f \simeq 0.15$, however, for $f>0.15$, the two data sets almost overlap. That is because the degree-BC relation of Eq. (1) holds up to roughly $g^{*} \simeq 8$ and breaks down beyond $g^{*}$, which corresponds to $k^{*} \simeq 15$. The fraction of vertices having degree $k>k^{*}$ is roughly $f \simeq 0.15$. We note that from the point of view of intentional attack, attack in BC order is more efficient than in degree order.

In conclusion, we have examined the $\mathrm{BC}-\mathrm{BC}$ correlation for the three types of scale-free networks, the dissortative, the neutral, and the assortative network. While the BC-BC correlation behaves similarly to the degree-degree correlation for the first two types, the BC-BC relation is nontrivial for the last type, and the mean $\mathrm{BC}$ of neighbors of a vertex with BC $g_{i}$ increases with increasing $g_{i}$ but very weakly, being almost independent of $g_{i}$. Such a behavior arises from the fact that the $\mathrm{BC}$ of the vertex with large degree is not always high, but takes rather widely ranged values.

The work is supported by the ABRL program of the KOSEF.
[1] R. Albert and A.-L. Barabási, Rev. Mod. Phys. 74, 47 (2002).

[2] S.N. Dorogovtsev and J.F.F. Mendes, Adv. Phys. 51, 1079 (2002).

[3] R. Albert, H. Jeong, and A.-L. Barabási, Nature (London) 401,
130 (1999)

[4] B.A. Huberman and L.A. Adamic, Nature (London) 401, 131 (1999).

[5] A.-L. Barabási and R. Albert, Science 286, 509 (1999). 
[6] M.E.J. Newman, Phys. Rev. Lett. 89, 208701 (2002); e-print cond-mat/0205405.

[7] M.E.J. Newman, e-print cond-mat/0209450.

[8] R. Kumar et al., in Proceedings of the 41st Annual Symposium on Foundations of Computer Science (IEEE Computer Society, Los Alamitos, 2000), p. 57.

[9] L.C. Freeman, Sociometry 40, 35 (1977).

[10] M.E.J. Newman, Phys. Rev. E 64, 016132 (2001).

[11] K.-I. Goh, B. Kahng, and D. Kim, Phys. Rev. Lett. 87, 278701 (2001).

[12] K.-I. Goh, E. Oh, H. Jeong, B. Kahng, and D. Kim, Proc. Natl. Acad. Sci. U.S.A. 99, 12583 (2002).

[13] P.L. Krapivsky and S. Redner, Phys. Rev. E 63, 066123 (2001).

[14] S.N. Dorogovtsev, J.F.F. Mendes, and A.N. Samukhin, e-print cond-mat/0206467.

[15] R. Pastor-Satorras, A. Vazquez, and A. Vespignani, Phys. Rev.
Lett. 87, 258701 (2001).

[16] The National Laboratory for Applied Network Research, sponsored by National Science Foundation (http://moat.nlanr.net).

[17] M. Molloy and B. Reed, Random Struct. Algorithms 6, 161 (1995); Z. Burda and A. Krzywicki, e-print cond-mat/0207020.

[18] Given a degree sequence $\mathcal{D} \equiv\left\{k_{i}\right\}(i=1, \ldots, N)$, we generate a maximally random network whose degree sequence is $\mathcal{D}$, with a constraint that any self-loops and multiple edges are forbidden. By choosing $\mathcal{D}=\left\{\left\lfloor(N / i)^{1 /(\gamma-1)}\right\rfloor\right\}$, we get a SF network with degree exponent $\gamma$, where $\lfloor\cdot\rfloor$ is the floor function. [19] http://www.imdb.com.

[20] A.-L. Barabási, H. Jeong, Z. Néda, E. Ravasz, A. Schubert, and T. Vicsek, Physica A 311, 590 (2002).

[21] M.E.J. Newman, Proc. Natl. Acad. Sci. U.S.A. 98, 404 (2001).

[22] R. Cohen, K. Erez, D. ben-Avraham, and S. Havlin, Phys. Rev. Lett. 86, 3682 (2001). 